\title{
Mechanical reinforcement and water repellency induced to cellulose sheets by a polymer treatment
}

\author{
Farouk Ayadi • Ilker S. Bayer • \\ Despina Fragouli • Ioannis Liakos • \\ Roberto Cingolani - Athanassia Athanassiou
}

Received: 10 October 2012/Accepted: 5 March 2013/Published online: 15 March 2013

(C) Springer Science+Business Media Dordrecht 2013

\begin{abstract}
The present study reports a simple method to control the mechanical and surface properties of cellulose fiber networks and to protect them from humidity, without altering their initial morphology. This is achieved by dip coating the fiber networks in solutions containing different amounts of ethyl cyanoacrylate monomer (ECA). Under ambient humidity and due to the presence of the -OH groups of the cellulose, the ECA polymerizes around each individual cellulosic fiber forming a thin poly(ethyl cyanoacrylate) (PECA) shell. PECA was found to interact with the cellulose surface via hydrogen bonding as evidenced by Fourier transform infrared spectroscopy and thermogravimetric analysis measurements. The detailed surface characterization reveals that only $3.5 \mathrm{wt} \%$ of ECA in solution is sufficient to form compact PECA cladding around every
\end{abstract}

Electronic supplementary material The online version of this article (doi:10.1007/s10570-013-9900-z) contains supplementary material, which is available to authorized users.

F. Ayadi · I. Liakos

Center for Biomolecular Nanotechnologies@Unile,

Istituto Italiano di Tecnologia (IIT), 73010 Lecce, Italy

I. S. Bayer · D. Fragouli · A. Athanassiou ( $\varangle)$

Nanophysics, Istituto Italiano di Tecnologia,

via Morego, 30, 16163 Genoa, Italy

e-mail: Athanassia.Athanassiou@iit.it

R. Cingolani

Istituto Italiano di Tecnologia, via Morego, 30,

16163 Genoa, Italy cellulose fiber. After the proposed treatment the cellulose sheets become hydrophobic, well protected from the environmental humidity and with increased Young's modulus.

Keywords Cyanoacrylate $\cdot$ Cellulose fibers · Hydrophobic cellulose $\cdot$ Relative humidity

\section{Introduction}

Paper is most commonly used in writing and printing support, made of a felt of cellulose fibers, held together by hydrogen bonds and sizing agents. Due to its abundance and low cost, use of cellulosic sheets in other applications such as packaging, electronics, sensors, medicine and so forth have become an attractive alternative to multifunctional materials (Barud et al. 2012; Kim et al. 2006; Zhenwen et al. 2011; Liang et al. 2012). In order to expand the uses of cellulose sheets, it is important to overcome the limitation of the hygroscopic properties of the cellulose, which lead to water absorption by the fibers and to the subsequent physical degradation of the material. For this reason, several approaches of surface modification of cellulosic fibers have been developed such as sizing, polymer grafting, cold plasma treatment, etc. (Li et al. 2010, 2012; Shen and Parker 2001; Zhang et al. 2007; Mukhopadhyay and Fangueiro 2009; Vaswani et al. 2005; Navarro et al. 2009; 
Samyn et al. 2011; Bongiovanni et al. 2011) in order to reduce their hydrophilic character and/or to improve their mechanical properties (Levy et al. 2002; Trombetta et al. 2005; Ye et al. 2009; Piantanida et al. 2006; Abdelmouleh et al. 2005; Deslandes et al. 1998). However, some of these methods have certain practical limitations because of one or more of the following reasons: They can be highly complex or/and expensive multi-step processes; they are not applied at the post-production stage; non-biodegradable, synthetic polymers are used posing environmental concerns; and finally, they modify the tactile sense and/or physical appearance of the final product. However, our group (Bayer et al. 2011; Fragouli et al. 2012) has recently demonstrated an approach to circumvent all of these drawbacks by applying a novel, simple and one-step method to obtain water-resistant multifunctional cellulose sheets by infusing them with ethyl cyanoacrylate (ECA) monomer solutions with or without functional micro/nano particles. Subsequent in situ polymerization of ECA monomer on the surface of the cellulose fibers forms a waterproof barrier around each fiber, which entraps the functional particles providing to the cellulose sheets their functionalities. This is a novel method which takes advantage of the adhesive property of cyanoacrylates. In fact, cyanoacrylate monomers are extensively used as tissue adhesives for the closure of skin wounds (Oowaki et al. 2000; Reece et al. 2001), and this property originates from the rapid polymerization that the cyanoacrylate undergoes by a nucleophilic attack reaction (Klemarczyk 2001) upon initiation by commonly occurring initiators such as moisture or free -OH groups on the surfaces at room temperature (Vauthier et al. 2003).

In this paper, we elucidate the type of interactions taking place between the cellulose fibers and the ECA monomer and we explain how the functional groups of cellulose initiate and affect the polymerization. The influence of the poly(ethyl cyanoacrylate) (PECA) cladding on the surface, thermal, and mechanical properties of the cellulose fiber networks is studied in detail, revealing the appropriate initial concentration of the ECA monomer solution needed for the full coverage and protection of each cellulosic fiber. We prove that the proposed treatment mechanically reinforces the cellulose sheets while their enhanced mechanical properties are fully preserved under different environmental humidity.

\section{Materials and methods}

\section{Materials}

Whatman Type- 1 cellulose sheets of $45 \mu \mathrm{m}$ thickness were used in all experiments unless otherwise noted. This product is chemically pure and free from silicones or other additives. No chemical or physical pretreatment was applied to the cellulose sheets before the experiments. Ethyl cyanoacrylate $\left(\mathrm{CH}_{2}=\mathrm{C}(\mathrm{CN}) \mathrm{CO}_{2} \mathrm{Et}\right)$ monomer and reagent grade toluene were purchased from Sigma-Aldrich and used as received.

Humidity conditioning and treatment with ECA

Before treatment with ECA, rectangular samples of Whatman paper (25 mm of length, $10 \mathrm{~mm}$ of width and approximately $0.045 \mathrm{~mm}$ thick) were conditioned under $80 \%$ relative humidity $(\mathrm{RH})$ for 4 days. At the end of this period the samples were dip coated with ECA solutions. To do this, different amounts of ECA were dissolved in toluene and vigorously mixed to obtain a wide range of different concentrations of ECA in solution. In particular ECA solutions of 1.0, 2.0, 3.5, 5.0, 10.0 and $15.0 \mathrm{wt} \%$ in toluene were prepared for dip coating.

For mechanical measurements using dynamic mechanical analysis, the following treatment procedure was followed: cellulose sheets were conditioned for 4 days in two different RH chambers (11 and $80 \%$ $\mathrm{RH})$ controlled by saturated salt solutions at $25^{\circ} \mathrm{C}$ before being dipped into ECA solutions for $15 \mathrm{~s}$ and left to dry under ambient conditions. Afterwards the treated cellulose sheets were stored for 4 days under 11 or $80 \%$ RH conditions before measurements. Pure PECA was prepared by drop casting ECA solution on silicon wafers under ambient humidity condition. Fourier transform infrared spectroscopy (FTIR) and thermogravimetric analysis (TGA) measurement of pure PECA were made on cross linked film samples obtained by peeling them off the silicon wafer substrates.

\section{Characterization}

Square samples (approximately $0.045 \mathrm{~mm}$ thick, $25 \mathrm{~mm}$ of length and $25 \mathrm{~mm}$ of width) were cut from the treated sheets to measure static water contact angle (WCA) ( $3 \mu \mathrm{L}$ of water droplets) using a contact angle 
goniometer (Kruss, Germany). The microscopy images of treated and untreated cellulose sheets were acquired by a polarized optical microscope (Carl Zeiss, Axio Scope A1) equipped with a digital camera and image processing software.

The mechanical properties of treated and untreated cellulose sheets were measured by a dynamic mechanical analyzer (DMA), T.A. Instruments, model 2980, with controlled force mode, using the clamp of tension film. The samples were approximately $10 \mathrm{~mm}$ wide, $0.045 \mathrm{~mm}$ thick, and the length between the clamps was measured by the DMA instrument. The set of experimental parameters used was: preload force, $0.005 \mathrm{~N}$; isothermal, at $25^{\circ} \mathrm{C}$; soak time, $1.0 \mathrm{~min}$; force ramp rate, $1 \mathrm{~N} \cdot \mathrm{min}^{-1}$ and upper force limit, $18.00 \mathrm{~N}$. Five measurements were conducted for each sample, and the results were averaged to obtain a mean value.

The decomposition of the paper samples were investigated by means of thermogravimetric analysis using a Mettler Toledo thermogravimetic/differential scanning calorimetry (TGA/DSC). Measurements were performed on 3-5 $\mathrm{mg}$ paper samples in an aluminum pan at a heating rate of $10{ }^{\circ} \mathrm{C} / \mathrm{min}$, from 30 to $600{ }^{\circ} \mathrm{C}$ in nitrogen atmosphere in order to determine the thermal stability of the cellulosic materials, as a function of ECA treatment. The weight loss (TG curve) and its first derivative (DTG curve) were recorded simultaneously as a function of time/ temperature.

X-ray photoelectron spectroscopy (XPS) measurements were conducted by a Specs Lab2 electron spectrometer equipped with a monochromatic X-ray source set at 1,253 eV and with a Phoibos analyzer Has 3500 (Hemispherical Energy Analyzer). The applied voltage of the $\mathrm{MgK} \alpha \mathrm{X}$-ray source was $13 \mathrm{kV}$ and the applied current $15 \mathrm{~mA}$. The pressure in the analysis chamber was approximately $7 \times 10^{-9}$ mbar. Large area lens mode was used for both wide and narrow scans. For the wide scan, the energy pass was $90 \mathrm{eV}$, the energy step was $0.5 \mathrm{eV}$ and the scan number was 1 . For the narrow high resolution scans, the energy pass was $30 \mathrm{eV}$ the energy step was $0.2 \mathrm{eV}$ and the scan number was 10 . The spectra were then analyzed using CasaXPS software.

The surface chemistry of the cellulose sheets was investigated by FTIR measurements in the $4,000-400 \mathrm{~cm}^{-1}$ spectral range using a Bruker Equinox 70 FT-IR apparatus in transmission mode at a resolution of $4 \mathrm{~cm}^{-1}$. All samples were stored at $80 \%$ RH before the FTIR analysis.

\section{Results and discussions}

After the dipping of the cellulosic sheets in the monomer solution, and during the air-drying step, the ECA polymerizes due to the presence of adsorbed ambient humidity on the fibers' surface (Tomlinson et al. 2006; Zhang et al. 2011), and due to the presence of the- $\mathrm{OH}$ groups of the cellulose, which act as polymerization initiators as well as terminators to the carbanionic propagating ends (Han et al. 2008). In fact, the $\mathrm{C}=\mathrm{C}$ double bond in ECA can be polarized in the presence of nucleophiles (water or carbanion) to form carbanions. In our previous works (Bayer et al. 2011; Fragouli et al. 2012), it was shown that the in situ generated PECA on cellulose fibers forms a cladding around each individual fiber and not an overall coating on the cellulose sheet. In fact, as shown in the polarized optical microscopy images of the untreated cellulose sheets (Fig. 1a) and sheets treated with $15.0 \mathrm{wt} \%$ of ECA in toluene solution (Fig. 1b), the overall fibers' morphology appears unaltered after treatment.

In order to understand whether or not any physicochemical interactions take place between the cellulose fiber surfaces and the PECA polymer, FTIR data were acquired from untreated and ECA treated cellulose fibers as shown in Fig. 2. Specifically, Fig. 2a shows the FTIR spectrum of the raw (untreated) cellulose paper, where all the characteristic absorption bands of cellulose is observed. In particular, the broad absorption band in the range of $3,700-3,100 \mathrm{~cm}^{-1}$ is attributed to the stretching of $\mathrm{H}$-bonded-OH groups; the $\mathrm{C}-\mathrm{H}$ stretching is observed at 2,800-3,000 $\mathrm{cm}^{-1}$ (Wang et al. 2007), and the peak at $1,615 \mathrm{~cm}^{-1}$ corresponds to the bending band of the adsorbed water (Cao and Tan 2004). Finally, the bands located in the region of $950-1,200 \mathrm{~cm}^{-1}$ are mainly due to $\mathrm{C}-\mathrm{O}$ stretching modes in alcoholic $\mathrm{C}-\mathrm{OH}$ moieties together with those of the intra and extracyclic C-O groups (Oh et al. 2005; Salmén and Bergström 2009). On the other hand, the FTIR spectrum of pure PECA (obtained from films deposited on silicon wafers) is shown in Fig. 2e, and is similar to previously reported studies (Zhai et al. 2009; Han et al. 2008; Arias et al. 2001). In fact, the 


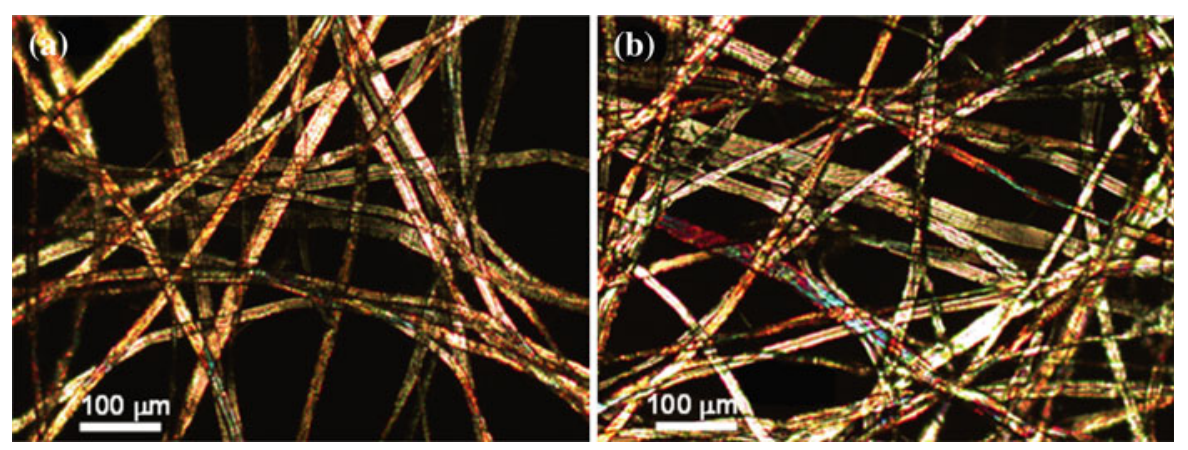

Fig. 1 Optical micrographs of $\mathbf{a}$ untreated and $\mathbf{b}$ treated cellulose fiber networks with $15.0 \mathrm{wt} \%$ ECA monomer in toluene

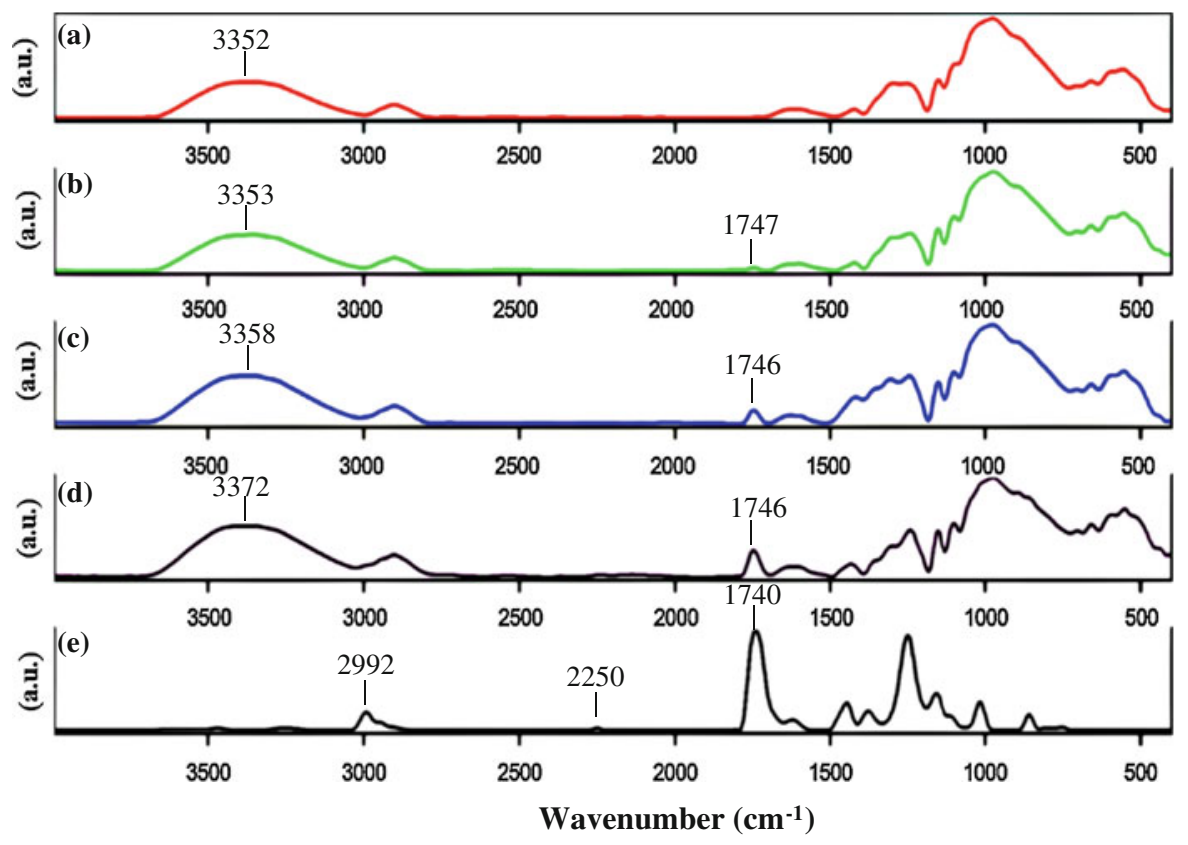

Fig. 2 FTIR spectra of a cellulose; cellulose treated with b $2.0 \mathrm{wt} \%$, c $10.0 \mathrm{wt} \%$, d $15.0 \mathrm{wt} \%$ of ECA in toluene; e PECA

characteristic absorption bands for the carbonyl $\mathrm{C}=\mathrm{O}$ ester $\left(1,746 \mathrm{~cm}^{-1}\right), \mathrm{C} \equiv \mathrm{N}$ groups $\left(2,250 \mathrm{~cm}^{-1}\right)$ and $\mathrm{C}-\mathrm{H}\left(2,890-3,050 \mathrm{~cm}^{-1}\right)$ are proofs of the polymerization of the ECA monomer (Tomlinson et al. 2006).

When cellulose sheets are treated with the ECA monomer solutions, the functional groups of both the cellulose and PECA can be identified in the FTIR spectra (Fig. 2b, c, d). In particular, it is observed that the stretching vibration of hydroxyl and carbonyl bands of the treated cellulose sheets shift towards higher wavenumbers compared to the neat polymers. For instance, the shift in the $-\mathrm{OH}$ and carbonyl group bands of cellulose sheet modified with $15.0 \mathrm{wt} \%$ of
ECA in toluene was measured to be approximately 20 and $6 \mathrm{~cm}^{-1}$, respectively. These shifts in bands indicate intermolecular interactions between cellulose and PECA. In fact, hydrogen-bonding interaction between carbonyl groups of PECA and hydroxyl group of cellulose would affect the local electron density and change the stretching vibration frequency of both groups and can give a rise to a shift (Kamińska and Sionkowska 1996; Chen et al. 2008). It is worth noting that pure PECA was polymerized under ambient humidity conditions while PECA deposited on the cellulose fibers was polymerized due to the presence of adsorbed ambient humidity on the fibers' 
surface and due to the presence of the $-\mathrm{OH}$ groups of the cellulose. This difference in PECA polymerization can be related to the shift of carbonyl band toward higher wavenumbers compared to that of neat PECA. In addition, since these shifts are towards higher wavenumbers, interactions between the functional groups of PECA and cellulose through hydrogen bonding are weaker than the chain-chain interaction within the pure cellulose and pure PECA, respectively (Marechal and Chanzy 2000).

Thermogravimetric analysis further reveals the existence of interactions between the cellulose fibers and the PECA shell. The treated cellulose sheets show two different steps of degradation, as seen in the DTG curves of Fig. 3 by one peak appearing below $180{ }^{\circ} \mathrm{C}$ and another one around $345^{\circ} \mathrm{C}$. The first peak is assigned to the decomposition of PECA and the second one is due to the degradation of the cellulose fibers as shown by the curves of the pure PECA and of the untreated cellulose of Fig. 3, respectively. Indeed, the decomposition of the pure cellulose fibers occurs at $348{ }^{\circ} \mathrm{C}$ and that of PECA at $192{ }^{\circ} \mathrm{C}$. As the ECA concentration in the solutions used for the cellulose treatment increases, the thermal signal related to the PECA degradation shifts towards higher temperatures $\left(165{ }^{\circ} \mathrm{C}\right.$ for $2.0 \mathrm{wt} \%, 170{ }^{\circ} \mathrm{C}$ for $10 \mathrm{wt} \%$ and $172{ }^{\circ} \mathrm{C}$ for $15 \mathrm{wt} \%$ ) approaching the one for pure PECA, while the decomposition temperature of the cellulose fibers decreases $\left(346{ }^{\circ} \mathrm{C}\right.$ for $2.0 \mathrm{wt} \%, 343{ }^{\circ} \mathrm{C}$ for $10.0 \mathrm{wt} \%$ and $342{ }^{\circ} \mathrm{C}$ for $15.0 \mathrm{wt} \%$ ) deviating from pure cellulose. This slight negative effect on the thermal stability of the cellulose after the ECA treatment can be attributed to the weakening of the inter-chain interactions of the fibers most likely due to the induced hydrogen bonding with the PECA, as previously discussed in the FTIR section.

However, the shift in PECA degradation temperature deposited on cellulose fibers towards the degradation temperature of the neat one when the ECA content is increased can be explained by the resulting thicker PECA cladding around the cellulose fibers. In fact, in such a case the initiation of ECA polymerization gets less affected by the hydroxyl groups of cellulose fibers, while it is more affected by the ambient humidity conditions, as in the case of the bulk PECA polymerized on silicon wafer.

In order to better understand the PECA shell formation on cellulose fibers as a function of the concentration of initial ECA solution, XPS

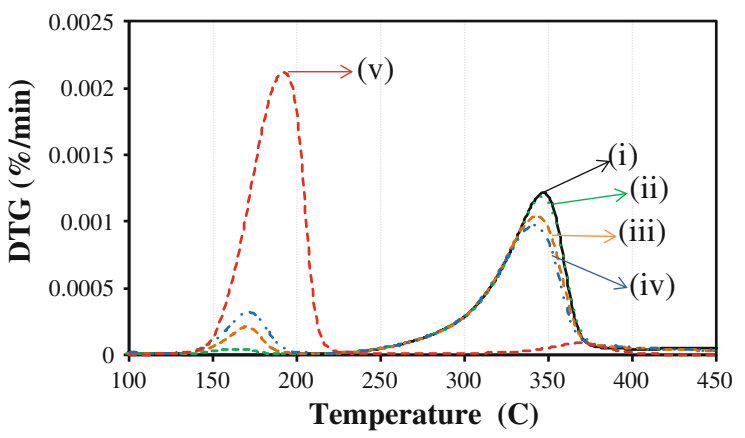

Fig. 3 DTG of (i) cellulose; cellulose treated with (ii) $2.0 \mathrm{wt} \%$, (iii) $10.0 \mathrm{wt} \%$, (iv) $15.0 \mathrm{wt} \%$ of ECA in toluene; (v) PECA

measurements were conducted, which help us to quantitatively study the chemistry of the outermost fiber surfaces. Figure 4 a shows the wide scan XPS results of pure cellulose and of cellulose fiber networks treated with toluene solutions of ECA with concentrations ranging from 1.0 to $15.0 \mathrm{wt} \%$. In all spectra, the carbon $(\mathrm{C})$ and oxygen $(\mathrm{O})$ peaks are present since these are characteristic species for both PECA and cellulose.

However the nitrogen peak $(\mathrm{N})$, characteristic for the PECA polymer, appears only in the treated cellulose sheets at $399.5 \mathrm{eV}$, proving, together with the $\mathrm{C} 1 \mathrm{~s}$ narrow scan analysis (see Figure $\mathrm{S} 1$ in the part 1 of Supporting information), the presence of PECA on the cellulose fiber surfaces. Most importantly, the amount of PECA indicated by the $\mathrm{N}$ content increases as the weight percent of ECA in the solution increases. In fact, as shown in Fig. $4 \mathrm{~b}$, the $\% \mathrm{~N}$ and the ratio (N/C) increase substantially with increasing ECA content before reaching a plateau at $3.5 \mathrm{wt} \%$ of ECA. This indirectly indicates that the specific concentration is sufficient to form a full, compact PECA coating on the fibers. Indeed as the XPS signal derives from the outermost layers of the fibers of thickness of few tens of nanometers, as soon as the PECA covers all the underlying cellulose fibers, the signal is expected to remain unaltered.

This observation is macroscopically confirmed by the wettability study of the treated cellulose sheets. Specifically, Fig. 5 shows the WCA measurements of the treated cellulose sheets as a function of ECA monomer content in the initial toluene solutions. As seen, the intrinsically water absorbing cellulose sheets become waterproof after the ECA treatment, while as the monomer amount increases, the fibers become 

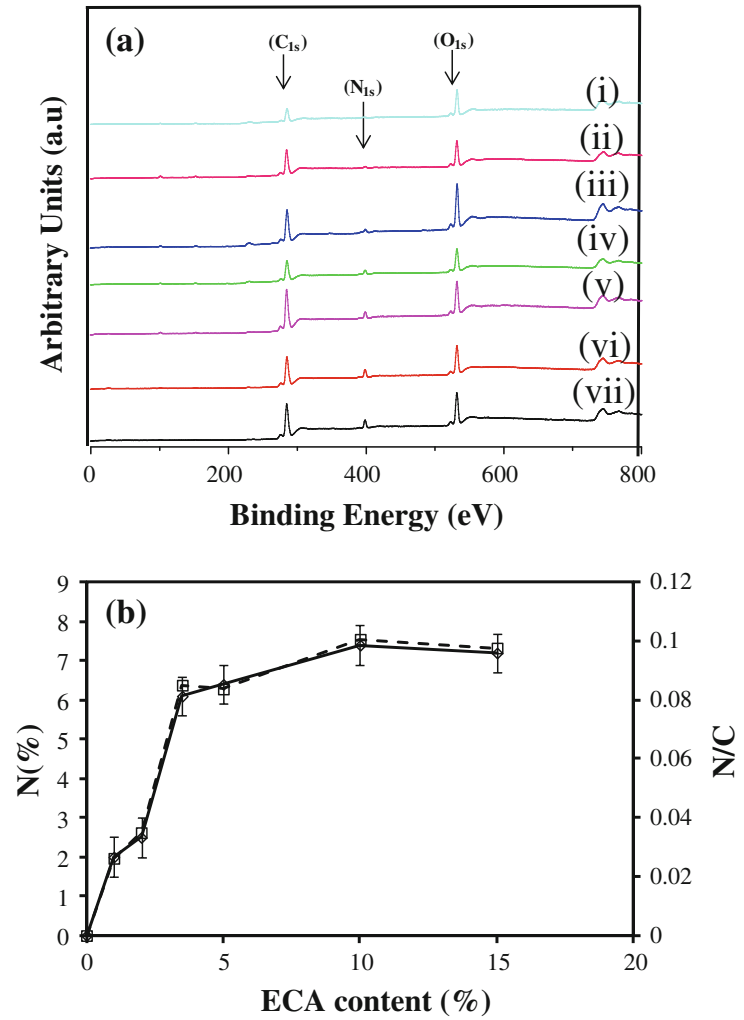

Fig. 4 a XPS wide scan results on (i) cellulose; cellulose treated with (ii) $1.0 \mathrm{wt} \%$, (iii) $2.0 \mathrm{wt} \%$, (iv) $3.5 \mathrm{wt} \%$, (v) $5.0 \mathrm{wt} \%$, (vi) $10.0 \mathrm{wt} \%$, (vii) $15.0 \mathrm{wt} \%$ of ECA; b Calculated percent nitrogen quantity (black line) and nitrogen to carbon ratio (N/C) (dashed black line) of the untreated and treated cellulose fiber networks as a function of ECA content from XPS wide scans

more hydrophobic. In fact, from 0 to $2.0 \mathrm{wt} \% \mathrm{ECA}$ treatment, the WCA increases linearly, while between 2.0 and $3.5 \mathrm{wt} \%$ the slope of the increase changes before reaching a plateau above $3.5 \mathrm{wt} \%$. The WCA value at the plateau is around $125^{\circ}$. Below this concentration, the amount of ECA seems to be insufficient to form a full, compact PECA cladding around each individual fiber, allowing water to penetrate into exposed surface areas of hygroscopic cellulose resulting in lower WCAs. This remarkable enhancement in treated cellulose fibers' hydrophobicity can be explained by the interaction which takes place between the two polymers. As previously mentioned in the FTIR section, this interaction occurs between the functional hydrophilic group of cellulose $(\mathrm{O}-\mathrm{H})$ and/or the adsorbed humidity, and the carbonyl group of PECA, leading to the reduction of the number of hydroxyl groups present on the surface of the

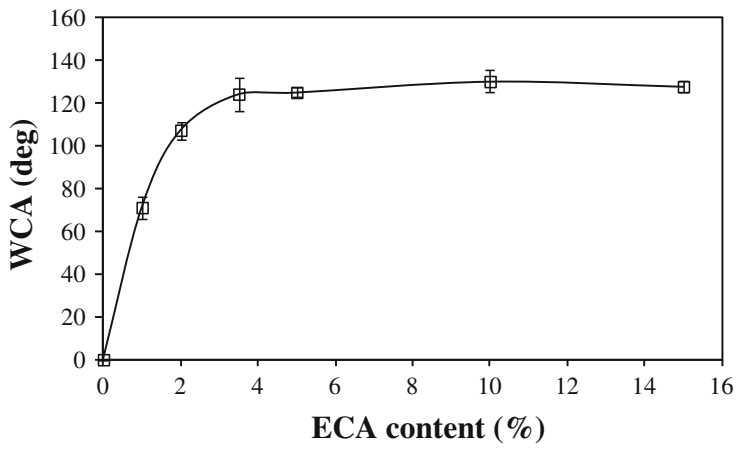

Fig. 5 WCA of treated cellulose as a function of ECA content in the toluene solutions used for the dip coating treatment

treated cellulose sheets, hence increasing the static WCAs. This is in accordance with Bayer et al. (2011) who also obtained waterproof PECA treated cellulose fibers. However, in that case the application of the ECA solutions to the cellulose fibers was different, and for this reason there are differences in the measured WCAs between these two studies on the treated fibers for a given ECA concentration, designating that the way the ECA treatment is applied on the cellulosic sheets plays an important role on the final hydrophobicity. In particular, direct drop casting ECA solutions with the same concentration, on the cellulose sheets did not display such high WCAs compared to the present results where the application is done by dip coating. The comparison with the data obtained using the drop casting method is commented in the supporting information (part 2).

In addition to the significantly increased hydrophobicity, it is also possible to control the mechanical properties of the cellulose sheets using the ECA treatment. In particular, Fig. 6 displays the evolution of the tensile modulus as a function of the ECA treatment. As expected, the modulus of untreated cellulose sheet ( $0 \mathrm{wt} \%$ ECA) stored at low humidity $(11 \% \mathrm{RH})$ is higher than the one stored at high humidity ( $80 \% \mathrm{RH})$ conditions, since the increase of the water content in the cellulose fiber sheet due to increased humidity results in the deterioration of the mechanical properties, suggesting a plasticizing effect of moisture on cellulose fibers (Spence et al. 2010).

In order to better evaluate the moisture effects on the mechanical properties of the PECA treated cellulose sheets, the samples which were stored at 11 or $80 \% \mathrm{RH}$ before ECA treatment were conditioned at the same RH conditions after ECA treatment, prior to 


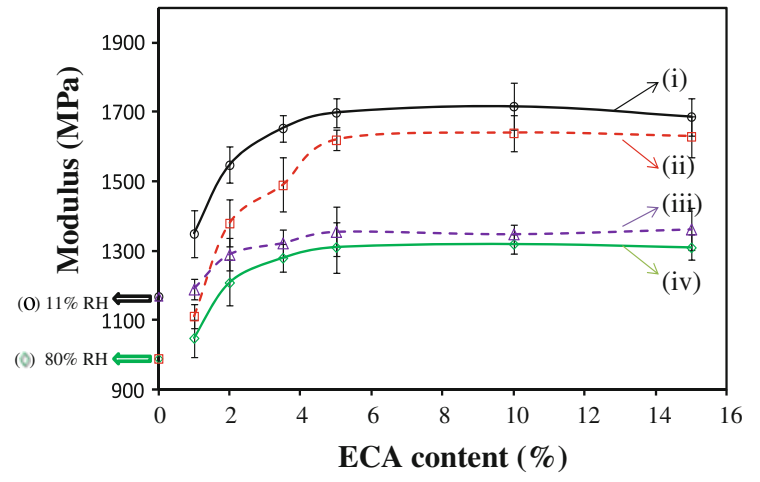

Fig. 6 Young's modulus variation of cellulose sheets as a function of the amount of ECA treatment under different initial and post humidity conditioning. (i) storage at $11 \% \mathrm{RH}$ before treatment and $11 \% \mathrm{RH}$ after treatment, (ii) storage at $11 \% \mathrm{RH}$ before treatment and $80 \% \mathrm{RH}$ after treatment, (iii) storage at $80 \% \mathrm{RH}$ before treatment and $11 \% \mathrm{RH}$ after treatment and (iv) storage at $80 \% \mathrm{RH}$ before treatment and $80 \% \mathrm{RH}$ after treatment

the DMA measurements. As Fig. 6 clearly demonstrates, independently from the humidity storage conditions, the modulus of all the treated cellulose sheets shows an increase with increasing ECA concentration until about $3.5 \mathrm{wt} \%$. After this concentration the modulus reaches a plateau, remaining practically constant at the maximum tensile modulus. The initial increase in Young modulus indicates that the mechanical properties of the sheets are dominated by the properties of the PECA layer which covers each fiber, prevailing over the reduction of the inter-chain interaction between the cellulose fibers due to the induced hydrogen bonding with the PECA. The stabilization of the Young modulus values for ECA concentration in the toluene solution above $3.5 \mathrm{wt} \%$ is another proof of the formation of a complete, homogeneous PECA layer around each cellulose fiber above this concentration, as previously demonstrated by the XPS and WCA analysis.

Figure 6 also reveals the protection that the formed PECA cladding around the cellulose fibers provides against the water uptake. In fact, the modulus value 1,169 MPa, of cellulose samples stored at $11 \% \mathrm{RH}$ before treatment, reaches $1,717 \mathrm{MPa}$ when the samples treated with solutions of ECA content greater than $3.5 \mathrm{wt} \%$, get transferred to low humidity conditions (11\% RH) (black line). This value appears only slightly decreased, 1,640 MPa, when the treated samples are conditioned at high humidity (80\% RH) (red dashed line). For the sheets treated with solutions of ECA content smaller than $3.5 \mathrm{wt} \%$ or for the untreated sheets, the differences in the modulus are much higher between the samples conditioned at low and high humidity environment. Such results indicate that when the PECA covers the cellulose fibers partially $(\leq 3.5 \mathrm{wt} \%$ ECA) the moisture can still penetrate into the fiber network and act as a plasticizer. On the other hand, the RH conditioning after the full coverage of the cellulose fibers with PECA cladding ( $>3.5 \mathrm{wt} \%$ ECA) seem to have a minimal effect on the mechanical properties of the cellulose sheets, demonstrating the effective protection of the treated material by the moisture.

The behavior of the cellulose samples stored at high RH content of $80 \%$ before their coverage with PECA (modulus $990 \mathrm{MPa}$ ) is very similar. In fact we can observe minimal differences in their Young modulus, when their conditioning after treatment with solutions of $>3.5 \mathrm{wt} \%$ ECA content, occurs at low (1,349 MPa) or high (1,320 MPa) RH (blue dashed and green line, respectively). Also in this case the differences are enhanced but slightly for the samples treated with solutions of $\leq 3.5 \mathrm{wt} \%$ ECA concentration. These differences are smaller than those observed for the corresponding samples stored at low RH environment prior to treatment. This is probably due to the fact that the high humidity on the fibers would induce better ECA polymerization and thus more compact claddings also for low ECA concentrations. In summary, ECA treatment along with humidity conditioning allows fabrication of treated cellulosic sheets with tunable mechanical modulus. Such modified cellulosic sheets can withstand sudden changes in humidity conditions rendering them more suitable for prolonged environmental applications.

\section{Conclusions}

A simple, inexpensive and single-step method is demonstrated in order to control the mechanical properties, and render cellulose sheets moisture resistant. By dip coating cellulose sheets in ECA monomer solutions and allowing polymerization of the monomer under ambient conditions on the fiber surfaces, highly moisture and mechanically resistant cellulosic sheets were obtained. The process does not alter the overall micromorphology of the cellulose fibers and hence the initial appearance of the cellulose sheets. 
Detailed XPS, wetting (contact angle) and mechanical analyses revealed that the optimum ECA concentration in toluene needed to fully cover each individual cellulose fiber is $3.5 \mathrm{wt} \%$, while FTIR and TGA studies specified the physicochemical interactions between the polymer and cellulose fibers. Due to its low cost, biocompatibility and biodegradability as well as its minimal impact on the appearance and morphology of the treated sheets, the present work is considered as a novel and attractive alternative to other proposed methods.

\section{References}

Abdelmouleh M, Boufi S, Belgacem MN, Dufresne A, Gandini A (2005) Modification of cellulose fibers with functionalized silanes: effect of the fiber treatment on the mechanical performances of cellulose-thermoset composites. J Appl Polym Sci 98(3):974-984

Arias JL, Gallardo V, Gómez-Lopera SA, Plaza RC, Delgado AV (2001) Synthesis and characterization of poly(ethyl-2cyanoacrylate) nanoparticles with a magnetic core. J Control Release 77(3):309-321

Barud HS, Caiut JMA, Dexpert-Ghys J, Messaddeq Y, Ribeiro SJL (2012) Transparent bacterial cellulose-boehmite-epoxi-siloxane nanocomposites. Compos Part A Appl Sci Manuf 43(6):973-977

Bayer IS, Fragouli D, Attanasio A, Sorce B, Bertoni G, Brescia R, Di Corato R, Pellegrino T, Kalyva M, Sabella S, Pompa PP, Cingolani R, Athanassiou A (2011) Water-repellent cellulose fiber networks with multifunctional properties. ACS Appl Mater Interfaces 3(10):4024-4031

Bongiovanni R, Zeno E, Pollicino A, Serafini P, Tonelli C (2011) UV light-induced grafting of fluorinated monomer onto cellulose sheets. Cellulose 18(1):117-126

Cao Y, Tan H (2004) Structural characterization of cellulose with enzymatic treatment. J Mol Struct 705(1-3):189-193

Chen ZG, Mo XM, He CL, Wang HS (2008) Intermolecular interactions in electrospun collagen-chitosan complex nanofibers. Carbohydr Polym 72(3):410-418

Deslandes Y, Pleizier G, Poiré E, Sapieha S, Wertheimer MR, Sacher E (1998) The surface modification of pure cellulose paper induced by low-pressure nitrogen plasma treatment. Plasmas Polym 3(2):61-76

Fragouli D, Bayer IS, Di Corato R, Brescia R, Bertoni G, Innocenti C, Gatteschi D, Pellegrino T, Cingolani R, Athanassiou A (2012) Superparamagnetic cellulose fiber networks via nanocomposite functionalization. J Mater Chem 22(4):1662-1666

Han MG, Kim S, Liu SX (2008) Synthesis and degradation behavior of poly(ethyl cyanoacrylate). Polym Degrad Stab 93(7):1243-1251

Kamińska A, Sionkowska A (1996) Effect of UV radiation on the infrared spectra of collagen. Polym Degrad Stab 51(1):19-26
Kim J, Yun S, Ounaies Z (2006) Discovery of cellulose as a smart material. Macromolecules 39(12):4202-4206

Klemarczyk P (2001) The isolation of a zwitterionic initiating species for ethyl cyanoacrylate (ECA) polymerization and the identification of the reaction products between $1^{\circ} 2^{\circ}$ and $3^{\circ}$ amines with ECA. Polymer 42(7):2837-2848

Levy I, Nussinovitch A, Shpigel E, Shoseyov O (2002) Recombinant cellulose crosslinking protein: a novel papermodification biomaterial. Cellulose 9(1):91-98

Li X, Tian J, Shen W (2010) Progress in patterned paper sizing for fabrication of paper-based microfluidic sensors. Cellulose 17(3):649-659

Li H, Fu S, Peng L, Zhan H (2012) Surface modification of cellulose fibers with layer-by-layer self-assembly of lignosulfonate and polyelectrolyte: effects on fibers wetting properties and paper strength. Cellulose 19(2):533-546

Liang H-W, Guan Q-F, Zhu Z, Song L-T, Yao H-B, Lei X, Yu S-H (2012) Highly conductive and stretchable conductors fabricated from bacterial cellulose. NPG Asia Mater 4:e19

Marechal Y, Chanzy H (2000) The hydrogen bond network in I-beta cellulose as observed by infrared spectrometry. J Mol Struct 523:183-196

Mukhopadhyay S, Fangueiro R (2009) Physical modification of natural fibers and thermoplastic films for composites-a review. J Thermoplast Compos Mater 22(2):135-162

Navarro F, Dávalos F, González-Cruz R, López-Dellamary F, Manríquez R, Turrado J, Ramos J (2009) Sisal chemo-thermomechanical pulp paper with a strongly hydrophobic surface coating produced by a pentafluorophenyldimethylsilane cold plasma. J Appl Polym Sci 112(1):479-488

Oh SY, Yoo DI, Shin Y, Seo G (2005) FTIR analysis of cellulose treated with sodium hydroxide and carbon dioxide. Carbohydr Res 340(3):417-428

Oowaki H, Matsuda S, Sakai N, Ohta T, Iwata H, Sadato A, Taki W, Hashimoto N, Yoshito I (2000) Non-adhesive cyanoacrylate as an embolic material for endovascular neurosurgery. Biomaterials 21(10):1039-1046

Piantanida G, Pinzari F, Montanari M, Bicchieri M, Coluzza C (2006) Atomic force microscopy applied to the study of Whatman paper surface deteriorated by a cellulolytic filamentous fungus. Macromol Symp 238(1):92-97

Reece TB, Maxey TS, Kron IL (2001) A prospectus on tissue adhesives. Am J Surg 182(2, Supplement 1):S40-S44

Salmén L, Bergström E (2009) Cellulose structural arrangement in relation to spectral changes in tensile loading FTIR. Cellulose 16(6):975-982

Samyn P, Schoukens G, Vonck L, Stanssens D, Van Den Abbeele Henk (2011) How thermal curing of an organic paper coating changes topography, chemistry, and wettability. Langmuir 27(13):8509-8521

Shen W, Parker IH (2001) A preliminary study of the spreading of AKD in the presence of capillary structures. J Colloid Interface Sci 240(1):172-181

Spence K, Venditti R, Rojas O, Habibi Y, Pawlak J (2010) The effect of chemical composition on microfibrillar cellulose films from wood pulps: water interactions and physical properties for packaging applications. Cellulose 17(4): 835-848

Tomlinson SK, Ghita OR, Hooper RM, Evans KE (2006) The use of near-infrared spectroscopy for the cure monitoring of an ethyl cyanoacrylate adhesive. Vib Spectrosc 40(1):133-141 
Trombetta T, Iengo P, Turri S (2005) Fluorinated segmented polyurethane anionomers for water-oil repellent surface treatments of cellulosic substrates. J Appl Polym Sci 98(3):1364-1372

Vaswani S, Koskinen J, Hess DW (2005) Surface modification of paper and cellulose by plasma-assisted deposition of fluorocarbon films. Surf Coat Technol 195(2-3):121-129

Vauthier C, Dubernet C, Fattal E, Pinto-Alphandary H, Couvreur P (2003) Poly(alkylcyanoacrylates) as biodegradable materials for biomedical applications. Adv Drug Deliv Rev 55(4):519-548

Wang L, Han G, Zhang Y (2007) Comparative study of composition, structure and properties of Apocynum venetum fibers under different pretreatments. Carbohydr Polym 69(2):391-397

Ye L, Filipe CDM, Kavoosi M, Haynes CA, Pelton R, Brook MA (2009) Immobilization of $\mathrm{TiO}_{2}$ nanoparticles onto paper modification through bioconjugation. J Mater Chem 19(15):2189-2198

Zhai Y, Deng L, Lin X, Xiao L, Jin F, Dong A (2009) Methoxy poly(ethylene glycol)-b-poly(ethyl cyanoacrylate) copolymer nanoparticles as delivery vehicles for dexamethasone. Chin Sci Bull 54(17):2918-2924

Zhang H, Kannangara D, Hilder M, Ettl R, Shen W (2007) The role of vapour deposition in the hydrophobization treatment of cellulose fibres using alkyl ketene dimers and alkenyl succinic acid anhydrides. Colloids Surf A Physicochem Eng Asp 297(1-3):203-210

Zhang L, Zhao N, Li X, Long Y, Zhang X, Xu J (2011) A facile approach to superhydrophobic coating from direct polymerization of "super glue". Soft Matter 7(8):4050-4054

Zhenwen D, Pinghung W, Girish C, Babak Z (2011) Ferrofluidimpregnated paper actuators. J Microelectromech Syst 20(1):59-64 